functional limitations, but for which there is yet no cure. Evidence-based treatment recommendations state that all patients with hand OA should be offered patient education, hand exercises, and provision of assistive devices and orthoses. Pharmacological therapy is recommended as a symptom relieving supplement. The main indication for CMCJ surgery is pain and poor function, and such surgery should be considered only when other treatment has proven insufficient in relieving pain (1). Previous research has shown that high motivation is a significant predictor for deciding to undergo CMCJ surgery (2), but there is little knowledge regarding which factors that motivates patients for undergoing such surgery. Objectives: The objective of this study was to explore patient goals and motivation for surgery, and factors characterizing patients highly motivated for surgery. Methods: This cross-sectional study included 180 patients referred from their general practitioner for CMCJ surgical consultation. Goals for surgery were collected with an open-ended question and analysed by linking the content of each goal to domains in the International Classification of Functioning, Disability and Health coding system. Motivation for surgery was rated with a Numeric Rating Scale (NRS, $0-10,0=$ no motivation). Activity limitations was self-reported using the Measure of Activity Performance of the Hand (MAP-Hand, score 1 to 4, 1=no activity problems) and the Disabilities of the Arm, Shoulder and Hand questionnaire (QuickDASH; score $0-100,0=$ no disability). Factors that characterized patients highly motivated for surgery (NRS $\geq 8$ ) were explored with multivariate regression analyses.

Results: Mean age of participants was 63 years (SD 7.6) and $142(79 \%)$ were women. The most common goals for surgery were to reduce pain and improve arm and hand use. Fifty-six (31\%) of the patients were characterized as highly motivated for surgery. High motivation for surgery was strongly associated with more activity limitations (MAP-Hand; (OR 4.00, $p=0.008)$ ), living alone (OR 3.18, $\mathrm{p}=0.007$ ) and a young age (OR 0.94, $\mathrm{p}=0.002$ ).

Conclusion: Decisions on CMCJ OA surgery should be based on assessment and discussion of patients' life situation, hand pain, activity limitations and motivation and goals for surgery. According to the EULAR recommendations, previously received conservative and pharmacological treatment should also be evaluated. References:

[1] Kloppenburg, M., et al. (2018). "2018 update of the EULAR recommendations for the management of hand osteoarthritis." Ann Rheum Dis. 0; 1-9

[2] Gravas, E. M. H., et al. (2019). "Non-pharmacological treatment gap preceding surgical consultation in thumb carpometacarpal osteoarthritis - a cross-sectional study." BMC Musculoskelet Disord 20(1): 180.

Disclosure of Interests: None declared

DOI: 10.1136/annrheumdis-2020-eular.3968

\section{FRI0636-HPR COMPARING THE EFFECTS OF YOGA AND HOME EXERCISE PROGRAMS ON FUNCTIONAL STATUS IN CHILDREN WITH ENTHESITIS RELATED ARTHRITIS}

E. Yasar ${ }^{1}$, E. Kavlak ${ }^{2}$, S. Turkucar ${ }^{3}$, D. Bayraktar ${ }^{4}$, C. Acari ${ }^{3}$, E. Unsal ${ }^{3}$. ${ }^{1}$ Pamukkale University, Institute of Health Sciences, Department of Physical Therapy and Rehabilitation, Denizli, Turkey; ${ }^{2}$ Pamukkale University, School of Physical Therapy and Rehabilitation, Denizli, Turkey; ${ }^{3}$ Dokuz Eylul University, Faculty of Medicine, Department of Pediatrics, Divison of Pediatric Rheumatology, Izmir, Turkey; ${ }^{4}$ Izmir Katip Celebi University, Faculty of Health Sciences, Department of Physiotherapy and Rehabilitation, Izmir, Turkey

Background: Enthesitis-Related Arthritis (ERA), also referred as the juvenile ankylosing spondylitis, is a subtype of Juvenile Idiopathic Arthritis (JIA). However, ERA patients were reported as having higher pain levels, lower functional status and lower quality of life level compared to other JIA subtypes. Yoga is used as an exercise method in adult patients with rheumatic diseases and positive effects on physical and psychosocial symptoms were reported. However, no study was conducted related to yoga in pediatric population with rheumatic diseases.

Objectives: The aim of this study was to investigate effects of performing yoga or home exercises on functional status in children with ERA.

Methods: Twenty-one children with ERA were allocated into two groups as yoga group $(n=11)$ and home exercise group $(n=10)$. Yoga group performed yoga exercises supervised by a physiotherapist for twice a week, for eight weeks, while the home exercise group performed video-based home exercises for the same period. Pain in rest and activity (Numeric Rating Pain Scale), functional status (Six-Minute Walk Distance, Stair Climb Test), and quality of life (PedsQL Children and Parent Form) were evaluated at baseline and following eight weeks.

Results: The groups were similar regarding to physical characteristics, pain, functional status, and quality of life at baseline ( $p>0.05$, Table 1$)$. While, all the parameters (except PEDSQL Parent Form) in yoga group significantly improved at the end of the study $(p<0.05$, Table 2$)$, only stair climb test results significantly improved in home exercise group ( $p<0.05$, Table 2$)$.
Table 1. Comparison of the groups at baseline

\begin{tabular}{lccc}
\hline & $\begin{array}{c}\text { Yoga Group } \\
\text { (n:11) } \\
\text { Median (IQR 25/75) }\end{array}$ & $\begin{array}{c}\text { Home Exercise Group (n:10) } \\
\text { Median (IQR 25/75) }\end{array}$ & $\mathbf{p}^{*}$ \\
\hline Physical parameters & & & \\
Age (year) & $15.0(13.0 / 15.5)$ & $16.0(14.0 / 17.0)$ & 0.099 \\
BMI (kg/m ${ }^{2}$ ) & $20.7(18.3 / 23.1)$ & $21.2(19.9 / 22.0)$ & 0.756 \\
Pain & & & \\
Rest pain (score) & $0(0 / 5.0)$ & $1.0(0 / 3.0)$ & 0.705 \\
Activity pain (score) & $4.0(1.0 / 6.5)$ & $2.0(0 / 7.0)$ & 0.387 \\
Functional Status & & & 1.000 \\
Six-minute walk distance (m) & $600.0(552.5 / 664.5)$ & $626.0(556.0 / 650.0)$ & 1.000 \\
Stair climb test (sec) & $7.6(6.8 / 8.3)$ & $7.4(7.0 / 8.0)$ & 0.654 \\
Quality of Life & $13.0(10.0 / 23.5)$ & $12.5(6.0 / 20.0)$ & 0.387 \\
PedsQL Children (score) & $21.0(8.5 / 31.5)$ & $26,0(15.0 / 39.0)$ & \\
PedsQL Parent (score) & & & \\
\hline
\end{tabular}

*: Mann-Whitney U test; IQR 25/75: Interquartile Range 25/75, PedsQL: Pediatric Quality of Life Inventory, $p<0.05$

Table 2. Changes in the Groups

\begin{tabular}{|c|c|c|c|}
\hline & $\begin{array}{c}\text { Before } \\
\text { Median } \\
\text { (IQR 25/75) }\end{array}$ & $\begin{array}{c}\text { After } \\
\text { Median } \\
(\text { IQR } 25 / 75)\end{array}$ & $\mathrm{p}^{*}$ \\
\hline \multicolumn{4}{|l|}{ Yoga Group } \\
\hline \multicolumn{4}{|l|}{ Pain } \\
\hline Rest pain (score) & $0(0 / 5.0)$ & $0(0 / 2.5)$ & 0.039 \\
\hline Activity pain (score) & $4.0(1.0 / 6.5)$ & $0(0 / 1.5)$ & 0.012 \\
\hline \multicolumn{4}{|l|}{ Functional Status } \\
\hline Six-minute walk distance $(\mathrm{m})$ & $600.0(552.5 / 664.5)$ & $692.0(595.0 / 705.5)$ & 0.011 \\
\hline Stair climb test (sec) & $7.6(6.8 / 8.3)$ & $6.2(6.0 / 7.0)$ & 0.008 \\
\hline \multicolumn{4}{|l|}{ Quality of Life } \\
\hline PedsQL Children (score) & $13.0(10.0 / 23.5)$ & $9.0(4.0 / 17.0)$ & 0.021 \\
\hline PedsQL Parent (score) & $21.0(8.5 / 31.5)$ & $18.0(8.0 / 22.0)$ & 0.169 \\
\hline \multicolumn{4}{|l|}{ Home Exercise Group } \\
\hline \multicolumn{4}{|l|}{ Pain } \\
\hline Rest pain (score) & $1.0(0 / 3.0)$ & $0(0 / 3.0)$ & 0.715 \\
\hline Activity pain (score) & $2.0(0 / 7.0)$ & $2.0(0 / 7.0)$ & 0.892 \\
\hline \multicolumn{4}{|l|}{ Functional Status } \\
\hline Six-minute walk distance $(\mathrm{m})$ & $626.0(556.0 / 650.0)$ & $601.5(585.0 / 707.0)$ & 0.093 \\
\hline Stair climb test (sec) & $7.4(7.0 / 8.0)$ & $7.0(6.5 / 7.8)$ & 0.028 \\
\hline \multicolumn{4}{|l|}{ Quality of Life } \\
\hline PedsQL Children (score) & $12.5(6.0 / 20.0)$ & $8.5(7.0 / 18.0)$ & 0.475 \\
\hline PedsQL Parent (score) & $26.0(15.0 / 39.0)$ & $22.5(14.0 / 30.0)$ & 0.192 \\
\hline
\end{tabular}

*: Wilcoxon Signed Rank Test, IQR 25/75: Interquartile Range 25/75, PedsQL: Pediatric Quality of Life Inventory, $p<0.05$

Conclusion: Yoga seems promising for improving functional status in children with ERA compared to a home-based exercise program. Therefore, yoga can be implemented as an exercise intervention in rehabilitation programs in children with ERA.

Disclosure of Interests: None declared

DOI: 10.1136/annrheumdis-2020-eular.5397

\section{FRI0637-HPR INDIVIDUALISED EXERCISE INTERVENTION FOR HIP AND KNEE OSTEOARTHRITIS: A SYSTEMATIC REVIEW AND META-ANALYSIS OF RANDOMISED CONTROLLEDTRIALS}

K. Yaseen ${ }^{1,2,3}$, B. Kundakci ${ }^{1}$, S. L. Goh ${ }^{4}$, M. Doherty ${ }^{1,5,6}$, W. Zhang ${ }^{1,5,6}$, A. Abhishek ${ }^{1,5,6}$, M. Hall ${ }^{1,5,6,7}$. ${ }^{1}$ University of Nottingham, Academic Rheumatology Department, Nottingham, United Kingdom; ${ }^{2}$ King Abdulaziz University, Physical therapy, Jeddah, Saudi Arabia; ${ }^{3}$ University of Nottingham, Division of Physiotherapy School of Health Sciences, Nottingham, United Kingdom; ${ }^{4}$ University of Malaya, Sports Medicine Unit, Kuala Lumpur, Malaysia; ${ }^{5}$ Nottingham Biomedical Research Centre, National Institute for Health Research, Nottingham, United Kingdom; ${ }^{6}$ University of Nottingham, Arthritis Research UK Pain Centre, Nottingham, United Kingdom; ${ }^{7}$ University of Nottingham, Division of Physiotherapy school of Health Sciences, Nottingham, United Kingdom

Background: Osteoarthritis $(O A)$ is a leading cause of disability worldwide. Currently, exercise is recognised to be one of the core treatments for OA (NICE, 2014). Convincing evidence shows that exercise can have positive effects on pain and function in people with OA (Fransen et al., 2015). However, a standardised exercise regimen may not suit all patients and adherence to exercise is always an issue. Therefore, several international guidelines recommended individualisation of the exercise regimen according to individual patient characteristics (e.g. pain severity, personal goals and co-morbidities), as this may enhance take up and adherence, hence treatment effect of the intervention (Fernandes et al., 2013). 